\title{
LEAVES OF MARKOV LOCAL MINIMAL SETS IN FOLIATIONS OF CODIMENSION ONE
}

\author{
JOHN CANTWELL, LAWRENCE CONLON
}

\begin{abstract}
The authors continue their study of exceptional local minimal sets with holonomy modeled on symbolic dynamics (called Markov LMS [C- C 1]). Here, an unpublished theorem of $G$. Duminy, on the topology of semiproper exceptional leaves, is extended to every leaf, semiproper or not, of a Markov LMS. Other topological results on these leaves are also obtained.
\end{abstract}

\section{Introduction}

Let $M$ be a compact, orientable manifold, $\mathcal{F}$ a transversely orientable $C^{2}$ foliation of $M$ of codimension one. Each component of $\partial M$, if there are any, is to be a leaf.

Let $X$ be a local minimal set (LMS) of $\mathcal{F}$. That is, there is an open, connected, $\mathcal{F}$-saturated subset $U \subseteq M$ and $X$ is a minimal set of $\mathcal{F} \mid U$. A special class of such sets, called Markov LMS, was introduced in [C-C 1]. These are exceptional LMS such that the holonomy pseudogroup of the foliated set $(X, \mathcal{F} \mid X)$ is, in a certain sense, generated by a subshift of finite type (see $\$ 1$ ). We will see, in $\S 6$, that every such subshift can occur for suitable Markov minimal sets in suitable $C^{\infty}$-foliated 3 -manifolds.

Let $\mathcal{E}(L)$ denote the set of ends of a leaf $L \subset X$, a compact, totally disconnected metrizable space of ideal points of $L$ at infinity. Let $\mathcal{E}^{*}(L)$ denote the closed subspace of ends that are asymptotic to $L$. In case $X$ is a minimal set of $\mathcal{F}$ (i.e., we take $U=M$ ), it is clear that $\mathcal{E}(L)=\mathcal{E}^{*}(L)$.

The first author was partially supported by N.S.F. Contract DMS -8420322 , the second by N.S.F. Contract DMS - 8420956. The second author would also like to thank the Centre de Recerca Matématica de institut d'Estudis Catalans, Barcelona, under whose hospitality this paper was completed. 
Theorem 1. Let $X \subset M$ be a Markov LMS of $\mathcal{F}$ and let $L$ be any leaf of $\mathcal{F} \mid X$. Then $\mathcal{E}^{*}(L)$ is homeomorphic to the Cantor set.

G. Duminy has proven this for semiproper leaves in an arbitrary exceptional LMS (unpublished). It is unknown whether his result extends to all leaves of the LMS.

Definition. A leaf $L$ is resilient if it has a holonomy contraction and if $L$ itself is captured by this contraction.

Definition. A handle in $L$ is a compack, connected, nonseparating submanifold $H$ of codimension one, $\partial H=0$. The genus of $L$ is the maximal number of pairwise disjoint handles in $L$ that are linearly independent in $H_{*}(L ; \mathbb{R})$.

Theorem 2. Let $X \subset M$ be a Markov LMS of $\mathcal{F}$. Then $X$ contains exactly a countable infinity of resilient leaves. Furthermore, either genus $(L)=1$, for each resilient leaf $L \subset X$, the remaining leaves of $\mathcal{F} \mid X$ having genus 0 , or every leaf $L \subset X$ has infinite genus and every end $\epsilon \in \mathcal{E}^{*}(L)$ is a cluster point of handles.

It was shown in [C-C 1] that each semiproper leaf in $X$ is resilient, but that there are only finitely many such leaves.

\section{Markov LMS}

Let $m \geq 2$ be an integer and let $\mathcal{K}$ denote the Cantor set $\{1,2, \ldots, m\}^{N}$ with typical element $\iota=\left(i_{1}, i_{2}, \ldots\right)$. Let $P=\left(p_{i j}\right)$ be an $m \times m$ matrix with entries from the set $\{0,1\}$. For many choices of $P$, the set.

$$
\mathcal{K}_{P}=\left\{\imath \in \mathcal{K} \mid p_{i_{i j} i_{j+1}}=1,1 \leq j \leq \infty\right\}
$$

is also a Cantor set. In any case, the map $\sigma: \mathcal{K}_{P} \longrightarrow \mathcal{K}_{P}$, defined by

$$
\sigma\left(i_{1}, i_{2}, i_{3}, \ldots\right)=\left(i_{2}, i_{3}, \ldots\right)
$$

is called a subshift of finite type, or a topological Markov chain [Wa]. A subshift of finite type is locally a homeomorphism. The psetudogroup $\Gamma_{\sigma}$ on $\mathcal{K}_{P}$, generated by the local one-one restrictions of $\sigma$, is said to be generated by the subshift $\sigma$.

If $X \subset M$ is an exceptional LMS for $\mathcal{F}$, fix $T \subset R$, a finite union of open, bounded intervals, let $\bar{T}$ denote the closure of $T$, a compact one-manifold, and fix an imbedding $\rho: \bar{T} \hookrightarrow U$, transverse to $\mathcal{F}$ and such that $C=X \cap \rho(T)$ is a Cantor set. Here, of course, $U$ is an open, connected, $\mathcal{F}$-saturated subset of $M$ such that $X$ is a minimal set for $\mathcal{F} \mid U$. Let $\Gamma$ denote the pseudogroup on $T$ induced by the holonomy of the foliation $\mathcal{F}$ and let $\Gamma \mid C$ be the induced pseudogroup on $C$. By an abuse, we suppress all mention of $\rho$ hereafter. 
Definition. Suppose that the above choices can be made so that there is a subshift of finite type $\sigma: \mathcal{K}_{P} \longrightarrow \mathcal{K}_{P}$ and maps $\tau: C \longrightarrow C$ and $\eta: C \longrightarrow \mathcal{K}_{P}$ having the following properties:

(1) $\tau$ is locally a homeomorphism and $\Gamma \mid C$ is generated by the local one-one restrictions of $\tau$;

(2) $\eta$ is a continuous surjection;

(3) $\eta^{-1}(\eta(x))$ is either a singleton or the pair of endpoints of a gap of $C$, for every $x \in C$;

(4) $\eta \circ \tau=\sigma \circ \eta$.

Then $\tau$ is said to be essentially a subshift of finite type generating $\Gamma \mid C, C$ is called a Markov $\Gamma$-minimal set, and $X$ is called a Markov LMS of $\mathcal{F}$.

The constructions of Sacksteder [Sa 1], Raymond [Ra], Hector [He], Ghys and Sergiescu [G-S], Inaba [In], and others provide examples of Markov minimal sets of a foliation $\mathcal{F}$. It is not hard to modify these to produce Markov LMS that are not minimal sets of $\mathcal{F}$.

Various elementary properties of Markov LMS were treated in [C-C 1, $\$ 1]$. For the sake of completeness, we review these briefly.

Definition. Let $S=\left(\left\{I_{1}, \ldots, I_{m}\right\},\left\{h_{1}, \ldots, h_{m}\right\}, P\right)$, where $P$ is an $m \times$ $m$ matrix of 0 's and 1 's, $I_{j} \subset R$ is a compact, nondegenerate interval, $h_{j}$ : $D\left(h_{j}\right) \longrightarrow R\left(h_{j}\right)$ is an orientation preserving homeomorphism between open, bounded intervals, and $I, \subset R\left(h_{j}\right), 1 \leq j \leq m$. Set $X_{j}=h_{j}{ }^{-1}\left(I_{j}\right)$. Let $\Gamma_{S}$ denote the pseudogroup generated by $\left\{h_{j}\right\}_{j=1}^{m}$. If the following properties hold, then $S$ is a Markov system and $\Gamma_{S}$ is a Markov pseudogroup:

(l) $R\left(h_{i}\right) \cap R\left(h_{j}\right)=0, i \neq j$;

(2) $p_{i j}=1$ implies that $I_{j} \subseteq X_{i}$;

(3) $p_{i j}=0$ implies that $I ; \cap D\left(h_{i}\right)=\emptyset$.

Assume that $S$ as above is a Markov system. Let $\left\{i_{1}, i_{2}, \ldots, i_{n}\right\} \subseteq\{1,2, \ldots$, $m\}$ and let $p_{i_{k} i_{k+1}}=1$, for $1 \leq k \leq n-1$. Then $w=h_{i_{1}} \circ \cdots \circ h_{i_{n}}$ is defined in $\Gamma_{S}$ and $X_{i_{n}} \subset D(w)$. For all such elements $w$, set $|w|=n$ and set $I_{w}=w\left(X_{i_{n}}\right)$. Finally, set

$$
\begin{aligned}
Z & =\bigcap_{n=1}^{\infty}\left(\bigcup_{|w|=n}^{\bigcup} I_{w}\right) \\
Z_{0} & =Z \backslash \operatorname{int}(Z) .
\end{aligned}
$$

For $x \in R\left(h_{i}\right)$, set $t(x)=h_{i}^{-1}(x) \in D\left(h_{1}\right)$. This well defines

$$
t: \bigcup_{i=1}^{m_{2}} R\left(h_{i}\right) \rightarrow \bigcup_{i=1}^{m} D\left(h_{i}\right)
$$

locally a homeomorphism, such that $t\left(Z_{0}\right) \subseteq Z_{0}$. Set

$$
\tau_{S}=t \mid Z_{0}: Z_{0} \longrightarrow Z_{0},
$$


a continuous map that is locally a homeomorphism, and remark that $\Gamma_{s} \mid Z_{0}$ is generated by $\tau_{\mathcal{S}}$.

Let $\iota=\left(i_{n}\right)_{n=1}^{\infty} \in \mathcal{K}_{P}$, and let $w_{n}$ be defined inductively as $w_{n-1} \circ h_{i_{n}}$. Then $I_{w_{n+1}} \subseteq I_{w_{n}}$ and the set $I_{t}=\bigcap_{n=1}^{\infty} I_{w_{n}}$ is either a singleton or a nondegenerate, compact interval. Let $\dot{I}_{t}$ denote the set-theoretic boundary of $I_{t}$, a set with one or two elements. It is clear that $Z=\bigcup_{\iota \in \mathcal{K}_{P}} I_{i}$ and $Z_{0}=\bigcup_{i \in \mathcal{K}_{P}} \dot{I}_{t}$. Define $\eta_{S}: Z_{0} \longrightarrow K_{P}$ by $\eta_{S}\left(\dot{I}_{2}\right)=\imath$ and remark that $\eta_{S} \circ \tau_{S}=\sigma \circ \eta_{S}$. Thus, we can say that $\tau_{S}$ is essentially a subshift of finite type.

1.1. Lemma. The set $K_{P}$ is a Cantor set and $\Gamma_{\sigma}$-minimal if and only if there exists a $\Gamma_{S}$-minimal Cantor set $C \subseteq Z_{0}$ such that $\eta_{S}(C)=K_{P}$. In this case $Z_{0} \backslash C$ is a union of at most countably many $\Gamma_{\mathcal{S}}$-orbits, each of which accumulates exactly on $C$.

For the elementary proof, see $[\mathrm{C}-\mathrm{C} 1,(1.1)]$. If, in fact, $\Gamma_{S}$ is of class at least $C^{2}$, it is true that $Z_{0} \backslash C$ is a union of at most finitely many $\Gamma_{\mathcal{S}}$-orbits, but this follows from a much more difficult theorem [C-C 1, (6.1)].

1.2. Lemma. Let $X$ be a Markov LMS of $\mathcal{F}$ and let $C, \Gamma, P, K_{P}, \tau$, and $\eta$ be as in the definition of such a set. Then this data can be chosen so that there exists a Markov sub-pseudogroup $\Gamma_{S} \subseteq \Gamma$, defined relative to the matrix $P$, such that $C \subseteq Z_{0}, \eta_{\mathcal{S}}\left|C=\eta: C \longrightarrow \mathcal{K}_{P}, \tau_{\mathcal{S}}\right| C=\tau$, and $\Gamma_{\mathcal{S}}|C=\Gamma| C$.

This was essentially proven in [C-C 1, (1.2)]. The relatively minor adjustments that are needed for the above formulation are left as an exercise.

Let $X \subset U$ be a Markov LMS. Thus $C=X \cap T$ is a Markov $\Gamma$-minimal set. We fix a Markov sub-pseudogroup $\Gamma_{S} \subseteq \Gamma$ as in (1.2).

Let $x \in C$ and let $\left|\Gamma_{S}(x)\right|$ denote the digraph of the orbit $\Gamma_{S}(x)$ relative to the set $\left\{h_{1}, \ldots, h_{n_{2}}\right\}$ of generators. Here, each positively oriented edge denotes an application of some $h_{i}, 1 \leq i \leq m$, hence moving backward along an edge denotes an application of $\tau$. By elementary symbolic dynamics, it is easy to see that this graph contains at most one cycle and that, if $v \in|\Gamma(x)|$ is a vertex not on a cycle, every positively directed edgepath out of $v$ of infinite length meets no cycle. Moreover, infinitely many vertices of such an edgepath are"branch points", in the sense that at least two distinct, positively directed, infinitely long edgepaths of $\left|\Gamma_{\mathcal{S}}(x)\right|$ emerge from each of them. These observations have certain easy consequences.

1.3. Lemma. The space $\mathcal{E}\left(\left|\Gamma_{S}(x)\right|\right)$ of ends of the graph $\left|\Gamma_{S}(x)\right|$ is a Cantor set.

We will prove Theorem 1 by showing that, under the hypotheses of that theorem, if $L_{x}$ is the leaf of $\mathcal{F} \mid X$ passing through $x \in T \cap X=C$, then there is a homeomorphism $\mathcal{E}\left(\left|\Gamma_{\mathcal{S}}(x)\right|\right) \cong \mathcal{E}^{*}\left(L_{x}\right)$. 
Definition. For $x \in C$, let $\Gamma_{x} \subset \Gamma$ denote the isotropy pseudogroup of $x$. The group

$$
\mathcal{H}_{x}\left(L_{x}, X\right)=\mathcal{H}_{x}(\Gamma(x), C)=\mathcal{H}_{r}\left(\Gamma_{S}(x), C\right)
$$

of germs at $x$ of elements $\gamma \in \Gamma_{x} \mid C$ is calied the holonomy group (at $x$ ) either of the leaf $L_{x}$ relative to $X$ or of the orbit $\Gamma(x)$ relative to $C$.

1.4. Lemma. The holonomy group $\mathcal{H}_{2}\left(\Gamma_{s}(x), C\right)$ of $\Gamma(x)$ relative to $C$ is either trivial or infinite cyclic and generated by a contraction that is unique in a suitable neighborhood of $x$ in $C$. The nontrivial cases are precisely the ones in which $\left|\Gamma_{\mathcal{S}}(x)\right|$ contains a cycle, the contraction being the holonomy produced by a positively directed loop around this cycle.

The proof is an exercise in elementary symbolic dynamics and, at any rate, will be found in [C-C 1, (3.2)].

1.5. Corollary. A leaf $L$ of $\mathcal{F} \mid X$ is resilient if and only if the holonomy group of $L$ relative to $X$ is infinite cyclic.

Our proof of Theorem 2 will show that the above holonomy contractions are compactly supported. Such a contraction determines a compactly supported cocycle on the leaf $L$ that is nontrivial in $H^{*}(L ; \mathbb{Z})$. That is, if the graph $\left|\Gamma_{\mathcal{S}}(x)\right|$ has a cycle, that cycle is Poincare dual to a handle on the leaf through $x$.

Remark that so far in this section we have not needed smoothness hypotheses. For the following, smoothness of class at least $C^{2}$ seems to be necessary.

1.6. Lemma. If $\mathcal{F}$ is of class $C^{2}$ and $X \subset M$ is a Markov LMS, then the Markov system $S$ of (1.2) can be chosen so that $I_{t}$ is a singleton, for each $\iota \in K_{P}$. In particular, $\eta: C \longrightarrow K_{P}$ is a homeomorphism.

Proof: We modify the Markov system $S$ of (1.2). If $I_{t}$ is a nondegenerate interval, then $[\mathrm{C}-\mathrm{C} 1,(3.4)]$ implies that $t=\left(J_{0}, J_{1}, J_{1}, \ldots, J_{1}, \ldots\right)$, where $J_{0}$ and $J_{1}$ are finite sequences of elements of $\{1,2, \ldots, m\}$. By applying a suitable nonnegative power of $T$ to $I_{l}$, we may assume that $J_{0}=\emptyset$. We set $J_{1}=\left(j_{1}, j_{2}, \ldots, j_{q}\right)$. If $I_{j_{k}}$ and $\tau^{k-1}\left(I_{l}\right)$ share an endpoint $\mathrm{x}$, delete $\{x\} \cup$ $\tau^{k-1}\left(\operatorname{int}\left(I_{t}\right)\right)$ from $I_{j_{k}}$. Otherwise, delete the interval $\tau^{k-1}\left(\operatorname{int}\left(I_{t}\right)\right)$ from $I_{j k}$. Similarly, delete $\{\tau(x)\} \cup \tau^{k}\left(\operatorname{int}\left(I_{t}\right)\right)$ or $\tau^{k}\left(\operatorname{int}\left(I_{t}\right)\right)$ from $X_{J_{k}}$. These operations should be performed for $1 \leq k \leq q$. This breaks each $I_{j_{k}}$ and $X_{j k}$ into a finite number of subintervals $I_{j_{k} 1}, \ldots, I_{j_{k} m_{k}}$ and $X_{j_{k}}, \ldots, X_{j_{k} m_{k}}$ (remark that possibly $j_{k}=j_{l}, k \neq l$ ). The generator $h_{j_{k}}$ of $\Gamma_{S}$ is then broken into $h_{j_{k} i}$ : $X_{j_{k} i} \longrightarrow I_{j_{k} i}, 1 \leq i \leq m_{k}, 1 \leq k \leq q$. It is elementary to check that this modification of $\mathcal{S}$ is again a Markov systern for the Markov LMS $X$. Finally, again because of the $C^{2}$ hypothesis, there arc only finitely many $\Gamma_{\mathcal{S}}$-orbits in $Z_{0}$ that are semproper $[\mathrm{C}-\mathrm{C} 1,(6.1)]$, so the above procedure only needs to be repeated finitely often to produce the desired Markov system. 
1.7. Corollary. If $\mathcal{F}$ is of class $C^{2}$, if $X \subset M$ is a Markov LMS, and if $\epsilon>0$ is given, then the Markov system $S$ of (1.2) can be modified to a Markov system $S^{*}$, for the same LMS $X$, such that the intervals $X_{i}^{*}$ and $Y_{i}^{*}$ are all of length less than $\epsilon$ and each generator $h_{i}^{*}$ is the restriction of a suitable generator $h_{j(i)}$ from $S$.

Proof: Apply $[\mathrm{C}-\mathrm{C} \mathrm{1},(4.1)]$ in conjunction with (1.6).

\section{The combinatorial arguments.}

The dynamical description of $\mathcal{F}$ is given via a suitable choice of open cover $\left\{U_{\alpha}\right\}_{\alpha \in \mathcal{A}}$ of $M$ by Frobenius coordinate charts. Each $\bar{U}_{\alpha}$ is to lie in the interior of some Frobenius chart and an $\mathcal{F}$-plaque in $U_{\alpha}$ is to meet at most one $\mathcal{F}$-plaque in $U_{\beta}$, for all $\alpha, \beta \in \mathcal{A}$.

The set of (closed) $\mathcal{F}$-plaques in $\bar{U}_{\alpha}$ can be identified with a compact, imbedded, $\mathcal{F}$-transverse arc $R_{0} \hookrightarrow M$, the preimage $R_{\alpha}$ being, itself, a compact subinterval of $R$. We arrange that these subintervals be disjoint in $\mathbf{R}$ with disjoint images in $M$. If $P_{\alpha} \in R_{\alpha}$ and $P_{\beta} \in R_{\beta}$ are interpreted as closed plaques, we will write

$$
\gamma_{\beta \alpha}\left(P_{\alpha}\right)=P_{\beta}
$$

if and only if $P_{\alpha}$ and $P_{\beta}$ overlap in the sense that

$$
\operatorname{int}\left(P_{\alpha}\right) \cap \operatorname{int}\left(P_{\beta}\right) \neq 0
$$

This defines the set of generators $G_{F}=\left\{\gamma_{\beta \alpha}\right\}_{\alpha, \beta \in A}$ of the holonomy pseudogroup $\Gamma_{\mathcal{F}}$ of $\mathcal{F}$, a pseudogroup on the compact one-manifold $R_{\mathcal{F}}=\bigcup_{\alpha \in \mathcal{A}} R_{\alpha}$. Thus, a word of length $n$ in these generators, when applied to a plaque $P \in R_{\mathcal{F}}$, amounts to a chain $\left(P=P_{0}, P_{1}, \ldots, P_{n}\right)$ of plaques such that $P_{i-1}$ and $P_{i}$ overlap, $1 \leq i \leq n$.

Let $\mathrm{X}$ be a Markov LMS of $\mathcal{F}$. We can assume that the imbedding $\bar{T} \leftrightarrow$ $U$, relative to which $X$ is of Markov type, is the imbedding of a compact submanifold $\bar{T} \subseteq R_{\mathcal{F}}$ induced by $R_{\mathcal{F}} \hookrightarrow M$.

By (1.2), there is a set $G_{S}=\left\{h_{i}\right\}_{1 \leq 1 \leq m}$, generating a Markov sub-pseudo group $\Gamma_{S} \subseteq \Gamma_{\mathcal{F}}$, such that $\Gamma_{\mathcal{F}}\left|C=\Gamma_{\mathcal{S}}\right| C$. Each $h_{i}$ is locally a composition of $\gamma_{\alpha \beta}$ 's. By (1.7), we can breal each $h_{i}$ up into finitely many of its restrictions to disjoint, open subintervals of $D\left(h_{i}\right)$, on each of which $h_{i}$ is a pure composition of $\gamma_{\alpha \beta}$ 's. Thus, $w \log$, we assume that each $h_{i}$ is a reduced word in the generators $G_{\mathcal{F}}$.

We must take into account the structure of the foliated manifold $(\widehat{U}, \widehat{\mathcal{F}})$ obtained by completing $(U, \mathcal{F} \mid U)$ relative to any Riemannian metric inherited from $M[\mathrm{Di}]$. Let

$$
\widehat{U}=K \cup V_{1} \cup \cdots \cup V_{r}
$$


be a decomposition into a compact nucleus $K$ (a foliated manifold with convex corners) and arms $V_{i}$ (foliated interval bundles), as described in [Di] (also, see $[\mathrm{C}-\mathrm{C} 2],[\mathrm{H}-\mathrm{H}],[\mathrm{Go}])$. Let $\mathcal{B} \subseteq \mathcal{A}$ be the subset such that $\bar{U}_{\alpha} \subset \operatorname{int}(K)$ if and only if $\alpha \in B$. Let $R=\bigcup_{\alpha \in \mathcal{B}} R_{\alpha}$.

The nucleus can be chosen large enough to engulf any specified compact subset of $U$. Thus, without loss of generality, we can assume that the Frobenius cover $\left\{U_{\alpha}\right\}_{\alpha \in \mathcal{A}}$ has been chosen so that $\bar{T} \subseteq R$ and the expressions for the generators $h_{\mathrm{i}}$ of $\Gamma_{\mathcal{S}}$ as words in the generators $\gamma_{\alpha \beta}$ of $\Gamma_{\mathcal{F}}$ involve only indices $\alpha, \beta \in \mathcal{B}$. Let $G=\left\{\gamma_{\alpha \beta}\right\}_{\alpha, \beta \in \mathcal{B}}$ and let $\Gamma$ be the pseudogroup on $R$ generated by the set $G$. Thus, $\Gamma_{s}|C=\Gamma| C$.

Definition. The foliated manifold $\left(M^{\prime}, \mathcal{F}^{\prime}\right)$ is defined by setting $M^{\prime}=$ $\bigcup_{\alpha \in \mathcal{B}} \bar{U}_{\alpha}$ and $\mathcal{F}^{\prime}=\mathcal{F} \mid M^{\prime}$. The subset $X^{\prime} \subset M^{\prime}$ is the $\mathcal{F}^{\prime}$-saturation of $C$.

Remark. Even if the foliated manifold $(M, \mathcal{F})$ has some degree of smoothness, $\partial M^{\prime}$ is only piecewise smooth, being divided by corners of various descriptions into smooth pieces that are each either transverse or tangent to $\mathcal{F}^{\prime}$.

In light of our discussion so far, the following is an exercise.

2.1. Lemma. The set $X^{\prime}$ is an exceptional minimal set of $\mathcal{F}^{\prime}$. Each leaf $L^{\prime}$ of $\mathcal{F}^{\prime} \mid X^{\prime}$ is contained in a unique leaf $L$ of $\mathcal{F} \mid X$ and the correspondence $L \leftrightarrow L^{t}$ is one-one between the leaves of $\mathcal{F} \mid X$ and of $\mathcal{F}^{\prime} \mid X^{\prime}$.

For $\delta \in \mathcal{B}$ and $x \in R_{\delta} \cap X^{\prime}$, we can choose $\gamma_{x} \in \Gamma$ to be a word of shortest length in the elements of $G$ such that $\gamma_{x}(x) \in C$. By compactness of $R_{\delta}$ and of $R_{\delta} \cap X^{\prime}$, there are finitely many compact subintervals with interiors covering $\operatorname{int}\left(R_{\delta}\right)$, each meeting $X^{\prime}$ in a set that lies entirely in the domain of some such $\gamma_{2}$ and is carried by $\gamma_{x}$ into $C$. That is, as $\delta$ ranges over $B$ and $x$ ranges over $R_{\delta} \cap X^{\prime}$, we can arrange that only finitely many $\gamma_{x}$ are distinct. Remark that, if $x \in C$, then $\gamma_{x}$ is an identity.

Finally, whenever $\alpha, \beta \in \mathcal{B}$ and $x \in D\left(\gamma_{\alpha \beta}\right) \cap X^{\prime}$, we can write

$$
\gamma_{\alpha \beta}(x)=y=\gamma_{y}^{-1} \circ w_{\alpha \beta}^{x} \circ \gamma_{x}(x)
$$

where $\gamma_{y}$ and $\gamma_{x}$ are as above and $w_{\alpha \beta}^{x}$ is a reduced word in the generators $G_{\boldsymbol{S}}$. Again, by compactness, there will only be finitely many distinct words $w_{\alpha \beta}^{x}$ that occur as $\alpha$ and $\beta$ range over $B$ and $x$ ranges over $D\left(\gamma_{\alpha \beta}\right)$.

Definition. For $w \in \Gamma_{S}$ a pure reduced word in the generators $G_{S}$, the length of that word is $\|w\|_{s}$. Similarly, for a pure reduced word $\gamma \in \Gamma$ in the generators $G$, the length is denoted by $\|\gamma\|$.

It is clear from the above discussion that we can fix a choice of $N \in \mathbf{Z}^{+}$that is simultaneously an upper bound to each of the following :

(a) $\left\|\gamma_{x}\right\|$, for each $x \in X \cap R$;

(b) $\left\|h_{j}\right\|, 1 \leq j \leq m$;

(c) $\left\|w_{\alpha \beta}^{\mathrm{x}}\right\|_{s}$, for each $x \in C$ and for all $\alpha, \beta \in \mathcal{B}$. 
Remark also that this implies

(d) $\left\|w_{\alpha \beta}^{x}\right\| \leq N^{2}$.

Let $L$ be a leaf of $\mathcal{F} \mid X, x \in L \cap T$, and let $L^{\prime}$ be the corresponding leaf of $\mathcal{F}^{\prime} \mid X^{\prime}$ as in (2.1). Then the graphs $|\Gamma(x)|$ and $\left|\Gamma_{S}(x)\right|$ will be defined relative to the generators $G$ and $G_{S}$, respectively, and so will be quite different. Also, since $\gamma_{\beta \alpha}=\gamma_{\alpha \beta}^{-1}$, we will agree that both $\gamma_{\beta \alpha}$ and $\gamma_{\alpha \beta}$ are to label the same edge with opposite directions. By contrast, each edge of $\left|\Gamma_{S}(x)\right|$ has been given a unique label $h_{j}$ and a corresponding preferred direction ( $c f . \S 1$ ).

The graph $|\Gamma(x)|$ is the 1-skeleton of the nerve of the plaque-cover of $L^{\prime}$, hence the first of the following lemmas is evident.

2.2. Lemma. The spaces $\mathcal{E}\left(L^{\prime}\right)$ and $\mathcal{E}(|\Gamma(x)|)$ are homeomorphic.

2.3. Lemma. The spaces $\mathcal{E}(|\Gamma(x)|)$ and $\mathcal{E}\left(\left|\Gamma_{\mathcal{S}}(x)\right|\right)$ are homeomorphic.

Proof: For $\alpha, \beta \in B$, let $z \in L \cap D\left(\gamma_{\alpha \beta}\right)$ and let $y=\gamma_{\alpha \beta}(z)$. Recall that

$$
\gamma_{\alpha \beta}(z)=\gamma_{y}^{-1} \circ w_{\alpha \beta}^{z} \circ \gamma_{z}(z)
$$

where $\left\|\gamma_{2}\right\|,\left\|\gamma_{y}^{-1}\right\|$, and $\left\|w_{\alpha \beta}^{*}\right\|_{s}$ are all bounded above by the integer $N$ and $\left\|w_{\alpha \beta}^{z}\right\|$ is bounded above by $N^{2}$.

The vertices of $\left|\Gamma_{\mathcal{S}}(x)\right|$ are contained in the set of vertices of $|\Gamma(x)|$ and this inclusion extends to a mapping $\left|\Gamma_{\mathcal{S}}(x)\right| \rightarrow|\Gamma(x)|$, not of graphs but of topological spaces. Indeed, an edge of $\left|\Gamma_{\mathcal{S}}(x)\right|$ labeled by $h_{j}$ is carried onto an edgepath in $|\Gamma(x)|$ corresponding to the expression for $h_{j}$ in terms of the generating set $G$.

The above mapping induces $\lambda: \mathcal{E}\left(\left|\Gamma_{\mathcal{S}}(x)\right|\right) \longrightarrow \mathcal{E}(|\Gamma(x)|)$, a continuous map. This is a surjection since every vertex of $|\Gamma(x)|$ can be joined to a vertex of $\left|\Gamma_{S}(x)\right|$ by an edgepath in $|\Gamma(x)|$ of length at most $\mathrm{N}$.

We must show that $\lambda$ is one-one. Let $\epsilon$ and $\epsilon^{\prime}$ be distinct elements of $\mathcal{E}\left(\left|\Gamma_{S}(x\}\right|\right)$. Let $\left\{x_{k}\right\}_{k=1}^{\infty}$ and $\left\{x_{k}^{\prime}\right\}_{k=1}^{\infty}$ be sequences of vertices of $\left|\Gamma_{S}(x)\right|$ converging to $\epsilon$ and $\epsilon^{\prime}$ respectively. Then, there is a vertex $x_{*}$ of this graph such that, for $k$ sufficiently large, any edgepath in $\left|\Gamma_{s}(x)\right|$ joining $x_{k}$ and $x_{k}^{\prime}$ must pass through $x_{*}$. Indeed, $x_{*}$ will be one of the points at which the graph "branches".

Let $D\left(x_{*}, \nu\right) \subset|\Gamma(x)|$ be the set of vertices that can be joined to $x_{*}$ by an edgepath of this graph of length at most $\nu$. In order to show that $\lambda(\epsilon) \neq \lambda\left(\epsilon^{\prime}\right)$, it will be sufficient to find an integer $\nu>0$ such that, for $k$ sufficiently large, every edgepath in $|\Gamma(x)|$ joining $x_{k}$ and $x_{k}^{\prime}$ must meet $D\left(x_{*}, \nu\right)$. For simplicity of notation, let $z=x_{k}$ and $z^{t}=x_{k}^{t}$. An edgepath joining these points corresponds to a word

$$
\gamma=\gamma_{\alpha_{q} \alpha_{q-1}} \circ \cdots \circ \gamma_{\alpha_{1} \alpha_{0}}
$$


such that $\gamma(z)=z^{\prime}$. Let $z(0)=z$ and

$$
z(p)=\gamma_{\alpha_{p} \alpha_{p-1}} \circ \cdots \circ \gamma_{\alpha_{1} \alpha_{0}}(z)
$$

$1 \leq p \leq q$. Since $z \in C$ and $z^{\prime}=z(q) \in C$, we see that $\gamma_{z}$ and $\gamma_{z^{\prime}}$ are identities, hence that

$$
\gamma(z)=w(z)=w_{\alpha_{q} \alpha_{q}-1}^{z(q-1)} \circ \cdots \circ w_{\alpha_{i} \alpha_{0}}^{z(\theta)}(z)
$$

Here, each $w_{\alpha_{i} \alpha_{i-1}}^{z(i-1)}$ is a word in the generators $G_{S}$, hence the above expression represents an edgepath in $\left|\Gamma_{\mathcal{S}}(x)\right|$ joining $z$ and $z^{\prime}$ and, as $s u c h$, passes through $x_{*}$. Let us say that $w_{\alpha_{p} \alpha_{p-1}}^{z(p-1)}$ represents a segment meeting $x_{*}$. It follows that $z(p) \in D\left(x_{*}, N^{2}+N\right)$.

Since endsets are compact Hausdorff spaces, we conclude that $\lambda$ is a homeomorphism.

2.4. Corollary. The space $\mathcal{E}\left(L^{\prime}\right)$ is homeomorphic to the Cantor set.

Proof: By (2.2) and (2.3), there is a homeonorphism

$$
\mathcal{E}\left(L^{\prime}\right) \cong \mathcal{E}\left(\left|\Gamma_{S}(x)\right|\right)
$$

and, by (1.3), the space $\mathcal{E}\left(\left|\Gamma_{s}(x)\right|\right)$ is a Cantor set.

2.5. Corollary. If $X$ is a Markov minimal set of $\mathcal{F}$, then the conclusion of Theorem 1 is true. (2.4).

Indeed, we can take $U=M=M^{\prime}, \mathcal{F}=\mathcal{F}^{\prime}, X=X^{\prime}, L=L^{\prime}$ and apply

2.6. Lemma. If $X \subset M$ is a Markov LMS of $\mathcal{F}$, then $X$ contains exactly a countable infinity of resilient leaves.

Proof: $\mathrm{By}(1.1), \mathcal{K}_{P}$ is a $\Gamma_{\sigma}$-minimal Cantor set. An elementary consequence is that exactly a countable infinity of $\left(i_{1}, i_{2}, \ldots, i_{k}, \ldots\right)$ are of the periodic form $(J, J, \ldots, J, \ldots) J$ being a finite string of integers from $\{1, \ldots, m\}$. By (1.4) and (1.5), the Markov LMS $X$ contains exactly a countable infinity of resilient leaves.

2.7. Lemma. Let $X \subset M$ be a Markov LMS of $\mathcal{F}$, let $L \subset X$ be a resilient leaf, and let $x \in L \cap T \subset C$. Then, the holonomy contraction that represents the generator of $\mathcal{H}_{x}\left(L^{\prime}, X^{\prime}\right)=\mathcal{H}_{x}(L, X) \cong Z$ is compactly supported on $L^{\prime}$.

Proof: We can assume that $x \in\left|\Gamma_{\mathcal{S}}(x)\right|$ lies on the unique cycle $w$ of that graph and we view $w$ as an edgeloop based at $x$. let $D(w, \nu) \subset|\Gamma(x)|$ denote the set of vertices that can be joined to a vertex of $w$ by an edgepath in this 
It is a consequence of Theorem 3 that, for a suitable (open) normal neighborhood $W$ of $F$ in $\hat{U}$, each component $\tilde{F}$ of $X \cap \bar{W}$ is diffeomorphic to $F_{\infty}$ in such a way that the projection $p: \widetilde{F} \longrightarrow F$, defined by projection along the normal fibers in $\bar{W}$, is identified with $\pi: F_{\infty} \longrightarrow F$. Here $\bar{W}$ will be diffeomorphic to the manifold $Q$ which is obtained from $F_{\sharp} \times[x, b]$ by the identification map

$$
\begin{gathered}
\varphi: H_{+} \times[x, b] \longrightarrow H_{-} \times[x, b], \\
\varphi\left(x_{+}, t\right)=\left(x_{-}, f(t)\right) .
\end{gathered}
$$

The manifold $Q$ is naturally foliated by leaves diffeomorphic to $F_{\infty}$ and the natural projection $\rho: Q \longrightarrow F$ restricts to $\pi$ on each of these leaves. The diffeomorphism $\bar{W} \cong Q$ carries each component of $X \cap \bar{W}$ onto one of these leaves and identifies $\rho$ with projection along the normal fibers of $\bar{W}$. This picture and the proof are, by now, standard in geometric foliation theory. For example, see $[\mathrm{C}-\mathrm{C} 2, \S 6]$ for the completely analogous situation of totally proper leaves winding in on leaves at lower levels. The situation there is precisely the one described here, but with $X$ replaced by a single leaf.

Let $F_{1}, \ldots, F_{q}$ be the components of $\partial \hat{U}$ on which $X$ accumulates, let $H_{1}, \ldots$, $H_{q}$ be the respective handles, and let $W_{1}, \ldots, W_{q}$ be the corresponding normal neighborhoods just described. Let $F_{q+1}, \ldots, F_{p}$ be the remaining components of $\partial \hat{U}$ and $W_{q+1}, \ldots, W_{p}$ respective normal neighborhoods of these in $\hat{U}$ that do not meet $X$. We can assume that, for $q+1 \leq j \leq p, \bar{W}_{j} \cap K$ is a finite union of closed Frobenius charts for $\hat{\mathcal{F}}$. Finally, let $M^{\prime}=K \backslash \bigcup_{1 \leq i \leq p} W_{i}$. By Theorem $3, \mathcal{F} \mid X$ has no holonony outside of $M^{\prime}$. At this point, the following is clear.

3.1. Claim. The Frobenius cover $\left\{U_{\alpha}\right\}_{\alpha \in A}$ for the foliated manifold $(M, \mathcal{F})$ can be chosen so that the manifold $M^{\prime}$, as described above, coincides with the manifold $M^{t}$ constructed in $\S 2$.

As in $\$ 2$, we set $\mathcal{F}^{t}=\mathcal{F} \mid M^{t}$, a foliation with exceptional minimal set $X^{t}$. Let $L$ be a leaf of $\mathcal{F} \mid X$ and let $L^{\prime}$ be the mique leaf of $\mathcal{F}^{\prime} \mid X^{\prime}$ contained in $L$ (2.1). Because the foliated manifold $\left(M^{\prime}, \mathcal{F}^{\prime}\right)$ has been fashioned with greater care than in $\S 2$, the leaf $L^{\prime}$ is a manifold with boundary, but with no corners. Indeed, $\left(M^{\prime}, F^{\prime}\right)$ is a foliated manifold with comers of various descriptons, and these comers divide $\partial M^{\prime}$, as usual, into a part tangent to $\mathcal{F}$, denoted $\partial_{\tau} M^{\prime}$, and a part transverse to $\mathcal{F}$, denoted $\partial_{\mathrm{N}} M^{\prime}$. The components of $\partial L^{\prime}$ are exactly the components of $L \cap \partial_{m} M^{\prime}$, each being a copy either of $H_{i}, 1 \leq i \leq q$, or of $\partial B_{j}, 1 \leq j \leq r$. There are infinitely many such components. Finally, each component of $L \backslash \operatorname{int}\left(L^{\prime}\right)$ has as boundary exactly one component of $\partial L^{\prime}$ and either lies entirely in $W_{i}, 1 \leq i \leq q$, or in some $V_{3}, 1 \leq j \leq r$.

3.2. Proposition. The inclusion $L^{\prime} \hookrightarrow L$ induces an imbedding $\lambda: \mathcal{E}\left(L^{\prime}\right) \hookrightarrow$ $\mathcal{E}(L)$ with image $\mathcal{E}^{*}(L)$. 
Proof: Since $\mathcal{E}\left(L^{\prime}\right)=\mathcal{E}^{*}\left(L^{\prime}\right)$, the image of $\lambda$ is contained in $\mathcal{E}^{*}(L)$. We must show that $\lambda$ maps $\mathcal{E}\left(L^{\prime}\right)$ one-one onto $\mathcal{E}^{*}(L)$.

Let $\epsilon$ and $\epsilon^{\prime}$ be distinct ends of $L^{\prime}$. Let $\Delta C \operatorname{int}\left(L^{\prime}\right)$ be a compact subset separating $\epsilon$ and $\epsilon^{\prime}$. Since the components of $L \backslash$ int $\left(L^{\prime}\right)$ correspond one-one to the components of $\partial L^{\prime}$, it is clear that $\Delta$, as a compact subset of $L$, also separates $\lambda(\epsilon)$ and $\lambda\left(\epsilon^{\prime}\right)$, hence these ends are distinct in $\mathcal{E}^{*}(L)$.

Let $\epsilon \in \mathcal{E}^{*}(L)$. Let $\cdots \subset U_{k} \subset U_{k-1} \subset \cdots \subset U_{1} \subset L$ be a fundamental system of neighborhoods of $\epsilon$. Since $\emptyset \neq U_{k} \cap M^{\prime}=U_{k} \cap L^{\prime}$ (the second equality is a consequence of (2.1)) we can choose $x_{k} \in U_{k} \cap L^{\prime}$. By passing to a subsequence, if necessary, we can assume that the sequence $\left\{x_{k}\right\}_{k=1}^{\infty}$ converges to an end $\eta \in \mathcal{E}\left(L^{\prime}\right)$. It follows that $\lambda(\eta)=\epsilon$.

\subsection{Corollary. Theorem 1 is trute.}

Proof: Apply (2.4) and (3.2).

3.4. Proposition. Let $L$ be a resilient leaf of $\mathcal{F} \mid X$. Then the holonomy contraction that represents the generator of $\mathcal{H}_{x}(L, X) \cong \mathbb{Z}$ is compactly supported on the leaf $L$.

Proof: As usual, the contraction on $L$ defines a nontrivial cohomology class $\theta \in H^{1}(L ; \mathbb{Z})$. If we view this class as a homomorphism

$$
\theta: \pi_{1}(L, x) \longrightarrow \mathbb{Z},
$$

we see, by the geometric consequences of Theorem 3 , that it vanishes on any loop that is freely homotopic to a loop on $L \backslash \operatorname{int}\left(L^{\prime}\right)$. Thus, $\theta$ is supported in $\operatorname{int}\left(L^{\prime}\right)$ where, by (2.7), it is compactly supported.

At this point, the proof of (2.8) applies without change.

3.5. Corollary. Theorem \& is true.

\section{The fundamental contraction.}

Let $X$ be an exceptional LMS, not necessarily of Markov type, and let $F$ be any proper or semiproper leaf of the foliation $\mathcal{F}$. By transverse orientability, $F$ has two sides, denoted $F^{+}$and $F^{-}$. Assume that $X$ is asymptotic to $F$ on at least one side, say on $F^{+}$. Let $0 \in F$ and let $[0, \epsilon]$ be a parametrized, $\mathcal{F}$-transverse arc, issuing from 0 on the side $F^{+}$. Let $] a, b[$ be a component of $|0, \epsilon| \backslash X$ such that $[a, b] \subset] 0, \epsilon \mid$

As in $\S 3, h_{\sigma}$ denotes holonomy in $\{0, \epsilon\}$ defined by a loop $\sigma$ on $F$, based at 0 , and $\sigma * H$ denotes the homological intersection number of $\sigma$ with a handle $H$.

Definition. Let $\sigma:[0,1] \longrightarrow L$ be a loop based at $\sigma(0)=\sigma(1) \in H$. If $\sigma \mid] 0,1[$ is in general position with respect to $H$, if $\sigma \mid] 0, \delta]$ and $\sigma \mid[1-\delta, 1[$ both lie on the same side of $H$ for small $\delta$, and if $\sigma$ always crosses $\mathrm{H}$ in the same direction, then we will say that $\sigma$ is in normal position with respect to $H$. 
4.1. Theorem.(Duminy). If $\epsilon>0$ is sufficiently small, then there is a handle $H \subset F$ and a loop $\sigma$ on $F$, based at 0 , such that $\sigma * H=1$ and $h_{\sigma}=f$ is a contraction of $[0, \epsilon]$ to 0 . Furthermore, for some integer $\kappa \geq 0$ and every loop $\tau$ on $F$, based at 0 and in normal position with respect to $H$,

$$
h_{\tau}\left(f^{k}(b)\right)=f^{k+r * H}(b)
$$

for each integer $k \geq k$, provided that $h_{\tau}\left(f^{*}(b)\right)$ is defined and $\leq b$.

Remark. If $F$ happens to be a semiproper leaf that lies in $X$ itself and if $F^{+}$is the nonproper side of $F$, this theorem leads rather easily to the result that $\mathcal{E}^{*}(F)$ contains no isolated points, hence is a Cantor set.

Let $U$ be an open, connected, $\mathcal{F}$-saturated set such that $X$ is a minimal set of $\mathcal{F} \mid U$. Let $F$ be a component of $\partial \hat{U}$ on which $X$ accumulates in $\widehat{U}$. Our main application of (4.1) will be to this situation, the side $F^{+}$being the side bordered by $U$, so $F^{+}$will be a proper side of $F$. The conclusion of (4.1) will be our starting point for the proof of Theorem 3 .

Unfortunately, Duminy has never made his proof of (4.1) available to the mathematical public. The result is crucial for current research into the structure of exceptional minimal sets, so we have prepared an account [C-C 3] for informal circulation.

Definition. The handle $H \subset F$, given by (4.1), will be called a holonomy handle.

\section{The proof of Theorem 3}

As in $\S 3$, the decomposition $\hat{U}=K \cup V_{1} \cup \cdots \cup V_{r}$ infruces a decomposition $F=A \cup B_{j_{1}} \cup \cdots \cup B_{j_{l}}$, where $F$ is a component of $\partial \hat{U}$. By suitably renumbering, we take $B_{j_{i}}=B_{i}, 1 \leq i \leq t$.

Let $x_{i} \in B_{i}$ and let $J_{i}$ be the fiber over $x_{i}$ of the interval bundle $V_{i} \rightarrow B_{i}$, $1 \leq i \leq t$. Let $P_{0}$ denote an $\mathcal{F}_{\text {-plaque containing the point } 0 \in A \text {. Edgeloops }}$ on $\left|\Gamma_{\mathcal{F}}(0)\right|$, based at 0 , induce a holonomy sub-pseudogroup $\Gamma_{F} \subset \Gamma_{\mathcal{F}}$, defined on open neighborhoods of 0 in $[0, \epsilon]$ and fixing 0 . The usual holonomy group $\mathcal{H}_{0}(F)$ is the group of germs at 0 of the elements of $\Gamma_{F}$.

We say that a chain $\rho=\left(P_{0}, P_{3}, \ldots, P_{4}\right)$ of plaques, without repetitions, is a simple chain at $P_{0}$, as is the holonomy element $h_{\rho} \in \Gamma_{\mathcal{F}}$ that it induces. A chain $\sigma=\left(P_{0}, P_{1}, \ldots, P_{q}, P_{0}\right)$ and the associated $h_{\sigma} \in \Gamma_{F}$ will be called a simple loop at $P_{0}$ if $\left(P_{0}, P_{1}, \ldots, P_{y}\right)$ is a simple chain. Finally, if $\rho=\left(P_{0}, \ldots, P_{q}\right)$ is a simple chain and $\sigma=\left(P_{q}, P_{q+1}, \ldots, P_{q+9}, P_{q}\right)$ is a simple loop at $P_{q}$, then

$$
\tau=\rho \sigma \rho^{-1}=\left(P_{0}, \ldots, P_{q}, P_{q+1}, \ldots, P_{q+s}, P_{q}, \ldots, P_{0}\right)
$$

and

$$
h_{r}=h_{\rho}^{-1} \circ h_{\sigma} \circ h_{\rho} \in \Gamma_{F}
$$


are called basic loops at $P_{0}$. Every clement of $\Gamma_{F}$, restricted to a suitable neighborhood of 0 in $[0, \epsilon]$, can be written as a composition of basic loops at $P_{0}$. Thus, the (germs of) basic loops generate $\mathcal{H}_{0}(F)$.

Let $A=A_{0} \subseteq A_{1} \subseteq \cdots \subseteq A_{k} \subseteq \cdots \subset F$ be an exhaustion of $F$ by compact, connected submanifolds with boundary. These should be chosen so that the components of $F \backslash A_{k}$ are not relatively compact in $F$ and so that these components correspond one-one to the components of $\partial A_{k}$. Let $G_{v}$ denote the set of basic loops at $P_{0}$ which, as plaque chains, consist entirely of plaques meeting $A_{0}$. Inductively, suppose that $G_{k}$ has been defined, some $k \geq 0$. Then $G_{k+1} \backslash G_{k}$ is to be the set of basic loops of the form $h_{\rho}^{-1} \circ h_{\sigma} \circ h_{\rho}$, where $\rho$ is a simple chain at $P_{0}$ that involves only placues that meet $A_{k}$ and contains just one plaque that meets $\partial A_{j}, 1 \leq j \leq k$, and $\sigma$ is a basic loop that involves only plaques that meet $A_{k+1} \backslash A_{k}$. View each $G_{k}$ as a subset of $\Gamma_{F}$ and let $G_{;}=\bigcup_{k=0}^{\infty} G_{k} \subseteq \Gamma_{F}$.

5.1. Lemma. For $\epsilon>0$ sufficiently small, and for ench $g \in G_{y}$, either $g$ or $g^{-1}$ is defined on $[0, \epsilon]$ and maps that interval into itself. Furthermore, $G_{b}$ generates $\mathcal{H}_{0}(F)$.

Proof: Since $G_{0}$ is finite, choose $\epsilon>0$ so small that both $g$ and $g^{-1}$ are defined on $[0, \epsilon]$ with images in $\left[0, \epsilon+\delta\left[\right.\right.$, for some $\delta>0$ and each $g \in G_{0}$. Either $g(\epsilon)$ or $g^{-1}(\epsilon) \in[0, \epsilon]$. Making $\epsilon$ smaller, if necessary, we make sure that $h_{\rho}$ is defined on $[0, \epsilon]$, for each simple chain $\rho$ at, $P_{0}$ in $A$. Since $V_{i}$ is a foliated interval bundle over $B_{i}, 1 \leq i \leq t$, it is true that either $g$ or $g^{-1}$ sends $[0, \epsilon]$ to itself, for each $g \in G_{1} \backslash G_{0}$.

The fundamental group $\pi_{1}(F, 0)$ can be defined via the nerve of the plaque cover of $F$ (provided the Frobenius cover of $M$ has been suitably chosen). Clearly the set of edgeloops in $\left|\Gamma_{F}(0)\right|$, corresponding to $G_{0}$, generates $\pi_{1}\left(A_{0}, 0\right)$. By the Van Kampen theorem and induction on $k$, the set of edgeloops, corresponding to $G_{k}$, generates $\pi_{1}\left(A_{k}, 0\right)$, hence those corresponding to $G_{\sharp}$ generate $\pi_{1}(F, 0)$. The natural surjection $\pi_{1}(F, 0) \longrightarrow \mathcal{H}_{0}(F)$ is then used to prove that $G_{i}$ generates $\mathcal{H}_{0}(F)$.

Definition. Let $g \in G_{\sharp} \backslash G_{k}$. The k-representation of $g$ is $h_{\rho}^{-1} \circ h_{\sigma} \circ h_{\rho}$, where $\rho$ is a simple chain in $A_{k}$ and $\sigma$ is a basic loop involving only plaques that meet $F \backslash A_{k}$.

Such a $k$-representation always exists and is unique. Its usefuness lies in the fact that, while $G_{q} \backslash G_{k}$ is generally an infinite set, the k-representations involve only finitely many of the simple chains $h_{\rho}$.

5.2. Lemma. Let $0<r<1<s$. Then there is an integer $k \geq 0$ such that, whenever $g \in G_{\sharp} \backslash G_{k}$ and $g=h_{\rho}^{-1} \circ h_{\sigma} \circ h_{\rho}$ is the $k$-representation, then $r<h_{\sigma}^{\prime}(x)<s$, for each $x \in h_{\rho}(D(g))$.

This lemma is just a slight variation on $[\mathrm{C}-\mathrm{C} 2,(2.9)]$, so we omit the proof. 
5.3. Lemma. If $\epsilon>0$ and $\delta>0$ are suitably chosen, each as small as desired, then every $g \in G_{\sharp}$ defines $g:[0, \epsilon] \longrightarrow[0, \epsilon+\delta[$. Furthermore, given $\eta>0, k$ can be chosen so large that $|g(u)-u|<\eta$, for each $g \in G_{\eta} \backslash G_{k}$, $0 \leq u \leq \epsilon$.

Proof: Let $\epsilon^{t}$ be as small as desired with the property ascribed to $\epsilon$ in (5.1). Let $\eta>0$. The generalized Kopell lemma $[\mathrm{C}-\mathrm{C} \mathrm{2,(2.8)]}$ is a consequence of (5.2) (cf. [C-C 2, p. 190]) and implies that, if $k$ is sufficiently large and if $g \in G_{\|} \backslash G_{k}$ maps $\left[0, \epsilon^{\prime}\right]$ into itself, then $|g(u)-u|<\eta, 0 \leq u \leq \epsilon^{t}$. By taking $\eta \leq \epsilon^{\prime} / 2$, we see that $g^{-1}$ maps $\left[0, \epsilon^{\prime} / 2\right]$ into $\left[0, \epsilon^{\prime}\right]$ and that

$$
\left|g^{-1}(u)-u\right|=\left|g^{-1}(u)-g\left(g^{-1}(u)\right)\right|<\eta
$$

$0 \leq u \leq \epsilon^{\prime} / 2$. That is, both $g$ and $g^{-1}$ send $\left[0, \epsilon^{\prime} / 2\right]$ into $\left[0, \epsilon^{\prime}\right]$ and satisfy

$$
|g(u)-u|<\eta>\left|g^{-1}(u)-u\right| \text {. }
$$

Since $G_{k}$ is finite, we can choose $\left.\epsilon \in\right] 0, \epsilon^{\prime} / 2[$ so small that $g$ sends $[0, \epsilon]$ into $\left[0, \epsilon^{t}\right]$, for each $g \in G_{k}$, hence for each $g \in G_{\sharp}$. Take $\left.\delta \in\right] \epsilon^{\prime}-\epsilon, \epsilon^{\prime}[$ and obtain all assertions.

5.4. Corollary. Let $0<r<\xi<s$. Then $\epsilon$ and $\delta$ can be chosen as in (5.9) and $k \geq 0$ can be chosen so that, for all $g \in G_{1} \backslash G_{k}$ and for $0 \leq u \leq \epsilon$, the inequalities $r<g^{\prime}(u)<s$ holl. If, furthermore, $\left\{g_{1}, \ldots, g_{n}\right\} \subseteq G_{k}$ is a subset such that no $g_{i}$ is germinally equivalent to a contraction to 0 , then the choice of $\epsilon$ can be made so small that $r<g_{i}^{\prime}(u)<s, 1 \leq i \leq n, 0 \leq u \leq \epsilon$.

Proof: By $\{5.2\}$, we choose $k \geq 0$ such that, for $g \in G_{\sharp} \backslash G_{k}$ with $k$ representation $g=h_{p}^{-1} \circ h_{\sigma} \circ h_{p}$, we have

$$
\sqrt{r}<h_{\sigma}^{i}\left(h_{\rho}(u)\right)<\sqrt{s},
$$

$0 \leq u \leq \epsilon$. By elementary calculus,

$$
g^{\prime}(u)=\frac{h_{\rho}^{\prime}(u) h_{o}^{\prime}\left(h_{\rho}(u)\right)}{h_{\rho}^{\prime}(g(u))}
$$

$0 \leq u \leq \epsilon$, and each of the finitely many functions $h_{\rho}^{\prime}$ is continuous. By making $\epsilon$ (and, if desired, $\delta$ ) small enough, we guarantee that

$$
\sqrt{r}<\frac{h_{\rho}^{\prime}(u)}{h_{\rho}^{\prime}(g(u))}<\sqrt{3}
$$

$0 \leq u \leq \epsilon$, hence

$$
r<g^{\prime}(u)<s,
$$


$0 \leq u \leq \varepsilon$. There remain the elements $g_{i} \in G_{k}, 1 \leq i \leq n$. Since none of these restricts to a contraction to 0 on any neighborhood of 0 in $[0, \epsilon]$, we see that $g_{i}^{\prime}(0)=1,1 \leq i \leq n$. Thus, by making $\epsilon>0$ possibly smaller, we complete the proof

At this point, we consider a Markov LMS, $X \subset M$, assumed to be a minimal set of $\mathcal{F} \mid U$ and to accumulate on $F \subseteq \partial \widehat{U}$ in $\hat{U}$. We let $|a, b|$ be a component of $j 0, \epsilon \mid \backslash X$, as in $\S 4$, and consider the holonomy handle $H \subset F$ and the holonomy contraction $f=h_{\sigma}$ given by (4.1). For each integer $n \geq 0$ and for $u \in D(f)$, we set $u_{n}=f^{n}(u)$.

5.5. Claim. Without loss of generality, we can assume that the $\mathcal{F}$-transverse 1-manifold $T$, as in $\xi 1$, is $] a_{1}, b_{0}\left[\right.$. This implies that $C \subset\left[b_{1}, a_{0}\right]$.

Proof: (1) We construct a holonomy imbedding $\psi: C \longrightarrow \mid a_{1}, b_{0}[$. If this has image $X \cap] a_{1}, b_{0}$, the first assertion follows. We will consider the contrary possibility in step (2).

If there is an integer $k \in\{1, \ldots, m\}$ such that $p_{2 k}=0,1 \leq i \leq m$, assume $w \log$ that $k=m$. The Markov system $S$ can be replaced by a Markov system $S^{\prime}$, indexed by $\{1, \ldots, m-1\}$, without loss of infomation. This replaces the subshift with one on a Cantor subset of $\{1, \ldots, m-1\}^{N}$ and replaces $C$ with a Cantor subset $C^{\prime}$. By finite repetition of this process, we assume that, for each index $k=1, \ldots, m$, there is an index $i \in\{1, \ldots, m\}$ with $p_{i k} \neq 0$.

From the above paragraph and elementary symbolic dynamics, it follows that the points $y \in C$ such that $\eta(y)$ is a periodic point in $\{1, \ldots, m\}^{\mathbb{N}}$ are dense in $C \cap I_{k}, 1 \leq k \leq m$. Since only finitely many periodic points can pertain to a semiproper $\Gamma$-orbit $[\mathrm{C}-\mathrm{C} 1,(6.2)]$, we choose $y_{k} \in C \cap I_{k}$ such that $C$ clusters on $y_{k}$ from both sides and $\eta\left(y_{k}\right)$ is periodic. Then there is a holonomy contraction $\theta_{k}$ of $I_{k}$ to $y_{k}$. Let $\varphi_{k}$ be an element of holonomy of $\mathcal{F} \mid U$ that carries $y_{k}$ to $\left.x_{k} \in\right] a_{1}, b_{0}\left[\right.$. It is easy to arrange that $x_{1}, \ldots, x_{k}$ all be distinct. For a suitably large integer $n$, $\psi_{k}=\varphi_{k} \circ \theta_{k}^{*}$ is defined on all of $I_{k}, 1 \leq k \leq m$, and $\left\{\psi_{k}\left(I_{k}\right)\right\}_{k=1}^{m}$ is a set of disjoint subintervals of $\mid a_{1}, b_{0}[$. Set

$$
\left.\psi=\bigcup_{k=1}^{m} \psi_{k} \mid\left(C \cap I_{k}\right): C \longrightarrow\right] a_{1}, b_{0}[
$$

the desired holonomy imbedcling. Clearly: $f(C)$ is an open-closed subset of $x \cap \mid a_{1}, b_{0} l$.

(2) We now assume that $C \subset \mid a_{1}, b_{0}[$. If $K=X \cap] a_{1}, b_{0} \mid \backslash C \neq \emptyset$, then $K$ is a Cantor set. For each $x \in K$, choose $\gamma_{x}$ as in $\$ 2$, a holonomy element with $\gamma_{2}(x) \in C$. As remarked in $\xi_{2}$, we can arrange that only finitely many $\gamma_{x}$ be distinct. Thus, $\left\{R\left(\gamma_{x}\right)\right\}_{x \in K}$ is actually a finite set of open intervals.

By (1.7), we can assume that the Markoy system $S$ is such that, for each $x \in K$ and $1 \leq i \leq m$, either $X_{t} \cap R\left(\gamma_{x}\right)=0$ or $X_{i} \subset R\left(\gamma_{x}\right)$. Select an open 
interval $U_{i}$ about each $X_{i}$ with these same relations to the intervals $R\left(\gamma_{i}\right)$. One then enlarges $S$ by adding the generators $\gamma_{x}^{-1} \mid U_{i}$ and the intervals $\gamma_{x}^{-1}\left(X_{i}\right)$. This Markov system generates the Cantor set $X \cap] a_{1}, b_{0}[$, so we can take $T=\left|a_{1}, b_{0}\right|$.

(3) Since $\left.] a_{1}, b_{1} \mid \cap X=\theta=\right] a_{0}, b_{0}\left[\cap X\right.$, it follows that $C \subset\left[b_{1}, a_{0}\right]$.

As in $\S 1, \Gamma$ denotes the pseudogroup on $T=\left|a_{1}, b_{0}\right|$ that is induced by $\Gamma_{\mathcal{F}}$. We have a Markov system

$$
\mathcal{S}=\left\{\left\{I_{1}, \ldots, I_{m}\right\},\left\{h_{1}, \ldots, h_{m}\right\}, P\right)
$$

and associated pseudogroup $\Gamma_{S} \subseteq \Gamma$, such that $\Gamma\left|C=\Gamma_{s}\right| C$. Since $C \subset\left[b_{1}, b_{0}[\right.$, we can take $I_{j} \subset\left\{b_{1}, b_{0}\left[, 1 \leq j \leq m\right.\right.$. Let $\Gamma_{\mathcal{S}}^{+}$denote the set of words in nonnegative powers of the generators $\left\{h_{1}, \ldots, h_{m}\right\}$.

We can assume that $H \subset \operatorname{int}(A)$, hence by cutting $A$ along $H$ we produce a compact, connected manifold $A^{\prime}$. It is fairly obvious how to select a subset of basic loops in $A$ that should be thought of as the basic loops on $A^{\prime}$. Let $G_{0}^{\prime}=\left\{g_{1}, \ldots, g_{m}\right\} \subset G_{0}$ be the elements of holonomy corresponding to the basic loops in $A^{\prime}$. Then, if $k \geq 0$ is sufficiently large, the set $\{f\} \cup G_{0}^{\prime}$ generates the same holonomy on $\left[0, b_{k}\right]$ as does $G_{0}$. By renumbering, replace $b_{0}$ with $b_{k}$. Thus, if $G_{\sharp}^{\prime \prime}=G_{0}^{\prime} \cup G_{1} \cup \cdots \cup G_{g} \cup \cdots$, we can assume that $G_{\sharp}=G_{\sharp}^{\prime} \cup\{f\}$.

In order to prove Therrem 3 , it should be clear that proving the following two propositions will suffice.

5.6. Proposition. For $k \geq 0$ sufficiently large, $G_{1}^{\prime}$ fixes every point of $X \cap\left[0, b_{k}\right]$.

5.7. Proposition. It is possible to choose the decomposition of $\widehat{U}$ so that the total holonomy of the foliated interval bundle $J_{i} \hookrightarrow V_{i} \longrightarrow B_{i}$ fixes every point of $X \cap J_{i}, 1 \leq i \leq t$.

Proof: (Using (5.6)) By [C-C 1, Theorem 1], each of the semiproper leaves $L \subset X$ has a holonony contraction on its nonproper side, say on $L^{+}$, and this contraction is unique relative to $X$. Apply (4.1) to the side $L^{+}$so as to conclude that this contraction is compactly supported. By [C-C 1, Theorem 2], there are only finitely many semiproper leaves in $X$, so we choose the customary decomposition $\hat{U}=K \cup V_{1} \cup \cdots \cup V_{r}$, in such a way that $K$ engulfs the holonomy handle on each of them.

Parametrize $J_{i}$ as $[0,1], 1 \leq i \leq t$. By $(5.6)$, the loops on $B_{i}$, based at $x_{i}$, fix every point of $X \cap[0, \delta]$, for some $\delta \in[0,1]$. The subset of $\left.X \cap\right] 0,1[$, fixed by these loops, is closed in $] 0,1[$, hence, if it is not all of $X \cap \mid 0,1[$, we can choose the above $\delta \in X \cap] 0,1\left[\right.$ in such a way that some loop on $B_{i}$ at $x_{i}$ induces a germinally nontrivial element of holonomy on $[\delta, \delta+\epsilon ! n X$ that fxes $\delta$. Again appealing to [C-C 1, Theorem 1], we see that, for $\epsilon>0$ sufficiently small, the holonomy around this loop gives a contraction of $[\delta, \delta+\epsilon[$ to $\delta$. 
But this loop also fixes each point of $[0, \delta] \cap X$ and, in a Markov LMS, each holonomy contraction to $\delta$ contracts a whole neighborhood of $\delta$ in $j 0,1[\cap X$ to $\delta$ (elementary symbolic dynamics). Thus, $\delta$ must lie on a semiproper leaf and this contradicts the fact that the holonomy handle on that leaf does not meet $V_{i}$.

In order to prove (5.6), we first review certain points from [C-C $1, \S 6]$, where the finiteness of the number of semiproper leaves in $X$ was established.

By the parametrization of $T$ as the subinterval $] a_{1}, b_{0}\left[\subset \mathbf{R}\right.$, we view $\Gamma_{S}$ as a pseudogroup on $\mathbf{R}$ and place the following definition.

Definition. A point $x \in \mathbb{R}$ is $\Gamma_{\mathcal{S}}^{+}$-uniform if there is an open neighborhood $V$ of $x$ in $\mathbf{R}$ and a number $\nu>0$ such that $g^{\prime}(u) / g^{\prime}(v) \leq \nu$, for each $g \in \Gamma_{\mathcal{S}}^{+}$ and for all $u, v \in V \cap D(g)$. The set of $\Gamma_{S}^{+}$-uniform points is denoted by $\mathcal{U}^{+}$ and its complement in $R$ by $\mathcal{B}^{+}$.

Remark. Since $\mathcal{U}^{+}$is open in $\mathbf{R}$ and, by default, contains $\left.\mathbf{R} \backslash\right] a_{1}, b_{0}[$, the set $\mathcal{B}^{+}$is compact.

In the following, $\tau$ again denotes the essential subshift that generates $\Gamma \mid C$. A $\tau$-cycle is a subset $\left\{x, \tau(x), \ldots, \tau^{k}(x)\right\} \subset C$ such that $k \geq 1$ and $\tau^{k}(x)=x$. The minimal such $k$ is the length of the $r$-cycle.

5.8. Lemma. The set $\mathcal{B}^{+} \cap C$ is the union of at most finitely many $\tau$ cycles. Furthermore, $\mathcal{B}^{+}$meets at most finitely many components of $T \backslash C$ and the points of $C$ bordering these components fall into finitely many $\tau$-cycles.

Proof: This was essentially proven in [C-C 1]. Indeed, by [C-C 1, (6.8), (6.18), and (6.19)] the set $\mathcal{B}^{+} \cap C$ and the set of components of $T \backslash C$ that meet $\mathcal{B}^{+}$are both finite. Let $x \in \mathcal{B}^{+}$and let $\mathrm{V}$ be a neighborhood of $x$ in $\mathbf{R}$. For each integer $N \geq 0$, let $W_{N}$ be the set of words of length $N$ in positive powers of $\left\{h_{1}, \ldots, h_{m}\right\}$. By $[\mathrm{C}-\mathrm{C} 1,(6.5)]$, we see that $\bigcup\left\{g(V \cap D(g)) \mid g \in W_{N}\right\}$ meets $\mathcal{B}^{+}$, for all choices of $V$ and $N$. From these facts the assertions follow easily.

Definition. The set $\mathcal{G}$ consists of all $g \in \Gamma$ such that

(a) $g$ maps $\left[b_{1}, b_{0}[\right.$ diffeomorphically onto itself;

(b) $g \mid C$ has fixed points $p<q$ with $g^{\prime}(p)=1=g^{\prime}(q)$ and such that $C \cap$ $|p, q| \neq 0$

(c) if $x \in C \cap] p, q\left[\right.$ and if $g(x)=x$, then $g^{\prime}(x) \neq 1$.

5.9. Lemma. There are constants $B>0$ and $0<r_{0}<1$ such that, if $g \in \mathcal{G}$ and $p, q$ are as in the above definition, there is a choice of $h \in\left\{g, g^{-1}\right\}$ and of $x \in] p, q[$ for which one of the following holds.

(1) $h(x)=x$ and $h^{\prime}(x)<r_{0}$.

(2) $p<h(x)<x$ and $(x-p) /(x-h(x)) \leq B$.

(3) $x<h(x)<q$ and $(q-x) /(h(x)-x) \leq B$. 
Proof: (1) Let $m_{i}=\min \left(C \cap I_{i}\right)$ and $M_{i}=\max \left(C \cap I_{i}\right), 1 \leq i \leq m$. These points border gaps of $C$, so we fix $n_{i}<m_{i}$ and $N_{i}>M_{i}$ in these gaps such that $n_{i}$ and $N_{i} \in R\left(h_{i}\right)$. Let

$$
\widetilde{B}=\max _{1 \leq i \leq m}\left\{\frac{N_{i}-m_{i}}{N_{i}-M_{i}}, \frac{M_{i}-n_{i}}{m_{i}-n_{i}}\right\} .
$$

The points $m_{i}$ and $M_{i}$ lie on semiproper $\Gamma_{\mathcal{S}}$-orbits, hence there are contractions $\gamma_{i}$ and $\eta_{i} \in \Gamma_{S}$ to $m_{1}$ and $M_{i}$, respectively, defined on the nonproper sides of these orbits and generating the infinite cyclic, relative holonomy groups $\mathcal{H}\left(\Gamma_{\mathcal{S}}\left(m_{i}\right), C\right)$ and $\mathcal{H}\left(\Gamma_{\mathcal{S}}\left(M_{i}\right), C\right)$ [C-C 1 , Theorem 1$]$.

Let $0<r_{0}<1$ be such that, for each value of $i=1, \ldots, m$, if $\gamma_{i}^{\prime}\left(m_{i}\right)$ and/or $\eta_{i}^{t}\left(M_{i}\right)$ are not equal to $\mathrm{I}$, then they are less than $r_{0}$. If all of these derivatives are 1 , then $0<r_{0}<1$ can be fixed arbitrarily.

(2) If $\mathcal{B}^{+} \neq 0$, let $\lambda$ be the length of the longest of the $\tau$-cycles given by (5.8) and let $\mu=\lambda^{2}$. If $\mathcal{B}^{+}=\emptyset$, $\mathrm{fx} \mu$ arbitrarily.

For $w=h_{i_{n}} \circ \cdots \circ h_{i_{1}} \in \Gamma_{S}^{+}$, let $w_{k}=h_{i_{2}} \circ \cdots \circ h_{i_{1}}, 0 \leq k \leq n$. Here we let $w_{0}=i d$.

Let $\mathcal{W}_{i}, 1 \leq i \leq m$, denote the set of all $w \in \Gamma_{S}^{+}$such that

(a) $I_{i} \subset D(w)$;

(b) either $|w|<\mu$ or $w_{n}\left(I_{i}\right) \cap B^{+}=\emptyset$.

By the definition of $B^{+}$, we can choose the points $n_{i}$ and $N_{i}$ of step (1) and a constant $\bar{B}>0$ such that $w^{\prime}(u) / w^{\prime}(v) \leq \bar{B}$, for each $w \in W_{i}$ and for all $u, v \in\left[n_{i}, N_{i}\right] \cap D(w), 1 \leq i \leq m$.

Finally, set $B=\tilde{B} \cdot \bar{B}$.

(3) Let $g \in \mathcal{G}$ and $p$, $q$ be as above. Since $C \cap|p, q| \neq \emptyset$, there is a unique shortest $w \in \Gamma_{S}^{+}$, possibly the identity, such that $[p, q] \subset D\left(w^{-1}\right), w^{-1}(p)=$ $p_{*} \in I_{i}, w^{-1}(q)=q_{*} \in I_{j}$, and $i \neq j$. Let $g_{*}=w^{-1} \circ g \circ w$ with $D\left(g_{*}\right)=\left\{p_{*}, q_{*}\right\}$.

Clearly $m_{j}$ and $M_{i}$ both lie in $\left[p_{*}, q_{*}\right] \subset D(w)$. Let $\bar{m}=w\left(m_{j}\right) \in[p, q]$ and $\vec{M}=w\left(M_{i}\right) \in[p, q]$. Since $p_{\star} \in I_{i}$ and $q_{*} \in I_{j}$ and $I_{1} \cap I_{j}=\emptyset$, it is also clear that

$$
\begin{aligned}
M_{i} & <N_{i}<q_{*}, \\
p_{*} & <n_{j}<m_{j},
\end{aligned}
$$

and we set $\bar{n}=w\left(n_{j}\right) \in[p, q], \bar{N}=w\left(N_{i}\right) \in[p, q]$.

(4) Suppose that either $\bar{m}$ or $\bar{M}$ is a fixed point of $g$. We will show that property (1) in the statement of the lemma follows.

For definiteness, suppose that $g(\bar{M})=\bar{M}$, the argument in the alternative case being completely parallel. There are two cases.

Case 1. Suppose that $p_{*}=M_{i}$, hence $p=\bar{M}$. Since $\left.C \cap\right] p, q[\neq \emptyset$ and since $M_{i}$ is the left endpoint of a gap of $C$, so is $\bar{M}=p$. We take $x \in|p, q|$ to be 
the other end of the gap. Then the fact that $w^{-3}(p)=p_{*}=M_{i}$ implies that $w^{-1}(x)=m_{k}$, some $k \in\{1, \ldots, m\}$. Also, $g(\vec{M})=\bar{M}$ implies that $g(x)=x$, while $x \in C \cap \mid p, q\left[\right.$ then implies that $g^{t}(x) \neq 1$. Either $g$ or $g^{-1}$ will be a contraction to $x$ and we choose $h=g^{ \pm 1}$ to be that contraction. Finally, let $h_{*}=w^{-1}$ o $h \circ w=g_{*}^{ \pm 1}$. Then $1>h^{\prime}(x)=h_{*}^{\prime}\left(m_{k}\right)$, so $h_{*}$ is a positive power of $\gamma_{k}$ and step (1) implies that $h^{\prime}(x)<r_{0}$, as desired.

Case 2. Suppose that $p_{*}<M_{i}$. Then, if we take $\left.x=v\left(M_{i}\right)=\bar{M} \in\right] p, q \mid$, we can argue exactly as in Case 1.

(5) By step (4), we can assume that neither $\bar{n}$ nor $\bar{M}$ is fixed by $g$. We will show that either property (2) or (3) in the statement of the lemma follows. We consider three possible cases.

Case 1. Suppose that $w \in \mathcal{W}_{i}$. Again clioosing $h=g^{ \pm 1}$ appropriately, we see that $h_{*}\left(N_{i}\right)<M_{i}$ and $h(\bar{N})<\bar{M}<\bar{N}$. From step (3), $\bar{M}$ and $\bar{N} \in[p, q]$, $w\left(M_{i}\right)=\bar{M}$, and $w\left(N_{i}\right)=\bar{N}$. By these remarks and the mean value theorem,

$$
\frac{\bar{N}-p}{\bar{N}-h(\bar{N})} \leq \frac{\bar{N}-p}{\bar{N}-\bar{M}}=\frac{w^{\prime}(\xi)}{w^{\prime}(\eta)} \cdot\left(\frac{N_{i}-p_{*}}{N_{2}-M_{i}}\right)
$$

with $\xi$ and $\eta$ both $\in \mid p_{*}, N_{i}\left[\right.$. Since $w \in \mathcal{W}_{i}$, we see that $w^{\prime}(\xi) / w^{\prime}(\eta) \leq \bar{B}$. Also, $N_{i}-p_{*} \leq N_{i}-m_{t}$, so

$$
\frac{\bar{N}-p}{\bar{N}-h(\bar{N})} \leq \ddot{B} \cdot\left(\frac{N_{i}-m_{i}}{N_{2}-M_{i}}\right) \leq \bar{B} \cdot \tilde{B}=B
$$

Thus, $x=\bar{N}$ satisfies property (2) in the statement of the lemma.

Case 2. Suppose that $w \in \mathcal{W}_{j}$. An argument completely parallel to that in Case 1 shows that $\bar{n}<h(\bar{n})<q$ and that $(q-\bar{n}) /(h(\bar{n})-\dot{n}) \leq B$, so we take $x=\bar{n}$, obtaining property (3) in the statement of the lemma.

Case 3. Suppose that $w \notin \mathcal{W}_{i}$ and $v \notin \mathcal{W}$. This cannot actually happen, as we now show.

We have $|w| \geq \mu$ and $w_{\mu}\left(I_{i}\right) \cap \mathcal{B}^{+} \neq \emptyset \neq w_{p}\left(I_{j}\right) \cap \mathcal{B}^{+}$. It follows from (5.8) that there are points $y_{i} \in I_{i}$ and $y_{j} \in I_{j}$, belonging to $r$-cycles of respective lengths $\lambda(i) \leq \lambda$ and $\lambda(j) \leq \lambda$, where $\lambda$ is as in step (2), and $w_{\mu}\left(y_{2}\right)$ and $w_{\mu}\left(y_{j}\right)$ belong to these same $\tau$-cycles. Let $k=\lambda(i) \lambda(j) \leq \lambda^{2}=\mu$. Let $g_{2}$ and $g_{j}$ be the words of lengths $\lambda(i)$ and $\lambda(j)$ fixing $y_{i}$ and $y_{j}$ respectively. It follows that

$$
w_{k}=g_{i}^{\lambda(j)}=g_{j}^{\lambda(i)}=h_{i_{k}} \circ \cdots \circ h_{i_{1}} .
$$

But then $y_{i}=w_{k}\left(y_{1}\right) \in I_{i_{k}}$, so $i_{k}=i$, while, similarly, $y_{j}=w_{k}\left(y_{j}\right) \in I_{i_{k}}$ and $i_{k}=j$. This contradicts the fact that $i \neq j$.

Proof of (5.6): Choose $\theta$ so that $f^{\prime \prime}(\xi) / f^{\prime}(\eta) \leq \theta$, for all $\xi$ and $\eta \in[0, \epsilon]$. Choose $r \in] r_{0}, 1\left[\right.$, sufficiently close to 1 that $\left(e^{\theta \varepsilon} B\right)^{-1}>1-r$. Here, of course, $r_{0}$ and $B$ are as in $(5.9)$. 
By (5.4), choose $k \geq 0$ so large that $g^{\prime}(x)>r$, for each $g \in G_{\sharp}^{\prime}, 0 \leq x \leq b_{k}$. We want to show that every $g \in G^{\prime}$ fixes every $x \in X \cap\left[0, b_{k}\right]$. If not, there exists $g \in G_{\Downarrow}^{\prime}$ and $n \geq k$ so that $g$ moves some points in $X \cap\left[b_{n+1}, b_{n}\right] \subset\left[0, b_{k}\right]$. Fix a choice of such $g$ and deduce a contradiction as follows.

Let $g_{0}=f^{-n} \circ g \circ f^{n} \in \Gamma$ and note that this carries $\left[b_{1}, b_{0}\right]$ diffeomorphically onto itself, fixing $b_{1}$ and $a_{0}=a$. Since the holonomy relative to $X$ of semiproper leaves in $X$ is compactly supported (4.1), we can assume that $k$ is so large that $g_{0}^{\prime}\left(b_{1}\right)=1=g_{0}^{\prime}\left(a_{0}\right)$. Thus, the closed set $C_{0}$ of $g_{0}$-fixed points $y \in X \cap\left[b_{1}, a_{0}\right]$ with $g_{0}^{\prime}(y)=1$ is nonempty. Since $g_{0}$ moves some points of $X \cap\left[b_{1}, a_{0}\right]$, there is a connected component $|p, q|$ of $\left[b_{1}, a_{0}\right] \backslash C_{0}$ such that $\left.X \cap\right] p, q[\neq \emptyset$. This proves that $g_{0} \in \mathcal{G}$ and we can apply (5.9).

If property (1) of (5.9) holks, then, for a suitable choice of an element $h=$ $g^{ \pm 1} \in G_{\sharp}^{\prime}$ and a corresponding choice of $h_{0}=g_{0}^{ \pm 1}$, we obtain the contradiction that $h^{\prime}\left(f^{n}(x)\right)=h_{0}^{\prime}(x)<r_{0}<r$, for some $\left.f^{n}(x) \in f^{n}\right] p, q\left[\subset\left[0, b_{k}\right]\right.$.

Thus, either property (2) or property (3) of (5.9) holds. We consider the first case, the second being entirely similar. Let $x \in|p, q|$ be such that inequalities $p<y=h_{0}(x)<x$ and $(x-p) /(x-y) \leq B$ hold. Set

$$
\begin{aligned}
& x_{n}=f^{n}(x) \\
& y_{n}=f^{n}(y)=h\left(x_{n}\right) \\
& p_{n}=f^{n}(p)=h\left(p_{n}\right) .
\end{aligned}
$$

By a standard calculation (cf. [Sa 2]),

$$
\frac{x_{n}-p_{n}}{x_{n}-y_{n}}=\frac{\left(f^{n}\right)^{\prime}(\xi)}{\left(f^{n}\right)^{\prime}(\eta)} \cdot\left(\frac{x-p}{x-y}\right) \leq \exp (\theta \epsilon) \cdot\left(\frac{x-p}{x-y}\right) \leq \exp (\theta \epsilon) \cdot B
$$

Then

$$
1-r<(\exp (\theta \epsilon) \cdot B)^{-1} \leq \frac{x_{n}-y_{n}}{x_{n}-p_{n}}=1-\frac{y_{n}-p_{n}}{x_{n}-p_{n}}=1-h^{\prime}(\zeta)
$$

so $h^{\prime}(\zeta)<r$ for suitable $\zeta \in\left[p_{n}, x_{n}\right] \subset\left[0, b_{k}\right]$, again a contradiction.

The proof of Theorem 3 is complete.

\section{Geometry of leaves}

Let $M$ be a closed, orientable 3 -manifold, $\mathcal{F}$ a transversely orientable $C^{2}$ foliation of $M$ by surfaces.

6.1. Conjecture. If $X \subset M$ is a Markov LMS of $\mathcal{F}$, and if $\bar{X}$ contains no toral leaf, then there is a Riemannian metric on $M$ relative to which every leaf of $\mathcal{F} \mid X$ has constant curvature -1 .

Indeed, refinements of the methods of this paper, together with standard facts about Teichmüller space, should yield a proof of (6.1) for the case of 
Markov minimal sets. At higher levels, further techniques from [C-C 4] will also be needed.

If $X$ is an arbitrary exceptional LMS of $\mathcal{F}$, it seems likely that a sequence $\left\{\mathcal{F}_{n}\right\}_{n=1}^{\infty}$ of foliations of $M$ can be found, converging to $\mathcal{F}$ in the $C^{2}$-topology, each with a Markov LMS $X_{n}$, such that $X$ is uniformly well $C^{2}$-approximated by $X_{n}$ as $n \rightarrow \infty$. This should lead to the following.

6.2. Conjecture. The previous conjecture is valid for an arbitrary exceptional LMS that does not approach a toral leaf.

This, together with the methods of [C-C 4], would give the following.

6.3. Conjecture. If no leaf of $\mathcal{F}$ is a torus or a sphere, and if no leaf is somewhere dense in $M$, then there is a Riemannian metric on $M$ relative to which every leaf of $\mathcal{F}$ has constant curvature -1 .

Indeed, we believe the following.

6.4. Conjecture. If no leaf of $\mathcal{F}$ is a torus, there exists a Riemannian metric on $M$ and a choice of $r \in\{-1,0,1\}$ such that every leaf of $\mathcal{F}$ has constant curvature $r$.

\section{References}

[C-C 1] J. CANTWELL AND L. CONLON, Foliations and subshifts, Tôhoku Math. J. 40 (1988), 165-187.

[C-C 2] J. Cantwell and L. Conlon, Poincaré-Bendixson theory for leaves of codimension one, Trats. Amer. Math. Soc. 265 (1981), 181-209.

[C-C 3] J. CANTweli AND L. Conlon, Endsets of exceptional leaves; a theorem of G. Duminy, (not intended for publication, but available upon request).

[C-C 4] J. CANTWELL AND L. CONLON, Leafwise hyperbolicity of proper foliations, to appear in Comment. Math. Helv.

[D] P. Dippolito, Codimension one foliations of closed manifolds, Ann. of Math. 107 (1978), 403-453.

[G-S] E. GHYS AND V. SERGiESCU, Sur un groupe remarquable de diffeomorphismes du cercle, Comment. Math. Helv. 62 (1987), 185-239.

[Go] C. Godbillon, "Feuilletages, Etudes Geometrique II," Publ. Inst. de Rech. Math. Avancée, Univ. Louis Pasteur, Strasbourg, 1986.

[He] G. HeCTOR, Quelques examples de feuilletages - especes rares, Ann. Inst. Fourier 26 (1975), 239-264. 
[H-H] G. HECTOR AND U. HIRSCH, "Introduction to the Geometry of Foliations, Part B," Vieweg, Braunschweig, 1983.

[In] T. INABA, Examples of exceptional minimal sets, preprint.

[Ra] B. RAYMOND, Ensembles de Cantor et feuilletages, Publ. Math. d'Orsay 191, 7656 .

[Sa 1] R. SACKSTEDER, On the existence of exceptional leaves in foliations of codimension one, Ann. Inst. Fourier 14 (1964), 221-226.

[Sa 2] R. SACKSTEDER, Foliations and pseudogroups, Amer. J. Math. 87 (1965), 79-102.

[Wa] P. WaLTERS, "An Introduction to Ergodic Theory," Graduate Texts in Math. 79, Springer-Verlag, New York, 1982.

Keywords. Foliation, leaf, Matkov chain, subshift, pseudogroup, exceptional local minimal set, endset, Cautor set, genus

J. Cantwell: Saint Louis University

St. Louis, MO 63103

USA

L. Conlon: Washington University

St. Louis, MO 63130

USA

Rebut el 3 de Novembre de 1988 